\title{
Termitofauna (Isoptera: Termitidae, Rhinotermitidae) en plantaciones de Pinus caribaea en sabanas de la Orinoquia Colombiana
}

\author{
Termites (Isoptera: Termitidae, Rhinotermitidae) in Pinus caribaea plantations in the Colombian Orinoco basin
}

\author{
MAIRA ALEXANDRA BELTRÁN-DÍAZ y OLGA PATRICIA PINZÓN-FLORIÁN²
}

\begin{abstract}
Resumen: Se estimó la composición de termitas asociada a plantaciones de Pinus caribaea de diferentes edades, establecidas en terrenos previamente ocupados por sabana seminatural y pastos artificiales, en la meseta de San Pedro en la Orinoquía colombiana (Villanueva, Casanare). Utilizando el protocolo del transecto, se muestrearon cuatro edades de plantación y áreas con pastos artificiales y sabana seminatural en dos épocas climáticas: húmeda 2013 y 2014 (marzo - abril) y seca de 2013 (noviembre - diciembre). La termitofauna encontrada estuvo compuesta de 28 especies pertenecientes a las familias Termitidae (Apicotermitinae, Nasutitermitinae, Termitinae, Syntermitinae) y Rhinotermitidae. Las plantaciones de siete años con poda y sin entresaca fueron las más diversas y la sabana seminatural alcanzó un mayor número de especies con respecto a los pastos introducidos, sin variaciones importantes en época de muestreo. La especie humívora Anoplotermes sp. 1 y la xilófaga Heterotermes tenuis fueron las más frecuentes en todas las edades de plantación, mientras en los otros usos del suelo el grupo más frecuente fue el humívoro con predominancia de Anoplotermes spp. Gracias al mejoramiento del microclima y al aumento de la disponibilidad de recursos alimenticios en plantaciones de pino de edades avanzadas, la diversidad y abundancia de termitas en este monocultivo es significativamente mayor que en pastos o sabanas, lo que sugiere que las termitas están interactuando con la dinámica natural de las plantaciones al contribuir en la descomposición del material celulósico y el mejoramiento de la fertilidad de los suelos.
\end{abstract}

Palabras clave: Xilófago, manejo forestal, usos del suelo.

\begin{abstract}
Termite abundance and diversity on Pinus caribaea stands established on lands previously occupied by a seminatural savanna and artificial grass were examined. Sampling was done during the transition from the dry to the rainy season in 2013 and 2014 (March - April) and the rainy to the dry season in 2013 (November - December) in plantations established in the Colombian Orinoco basin region, commonly known as "Mesa de San Pedro" (Villanueva, Casanare). Using the transect protocol, stands of Pinus caribaea of four different ages, and areas with introduced grass and seminatural savanna were sampled. Termite fauna was composed of twenty eight morphospecies belonging to the Termitidae (Apicotermitinae, Nasutitermitinae, Termitinae, Syntermitinae) and Rhinotermitidae (Heterotermitinae and Rhinotermitinae) families. Seven year-old pine stands without pruning or thinning had the most diversity, while natural savanna had a greater number of species than introduced grasses. No significant differences were observed between sampling seasons. The humivorous species Anoplotermes sp. 1 and the xylophagous Heterotermes tenuis were the most common in pine stands, while several species belonging to the humivorous genus Anoplotermes spp. predominated in pastures and savanna vegetation. Changes in the microclimate and increased availability of food resources in older pine stands may explain the higher diversity and abundance of termites in this monoculture as compared to pastures or savanna vegetation, suggesting that termites are interacting with the natural dynamics of plantations by contributing to the decomposition of cellulosic material and the improvement of soil fertility.
\end{abstract}

Key words: Xylophagous, forest management, land uses.

\section{Introducción}

Los artrópodos, en especial la macrofauna del suelo, se consideran excelentes indicadores de la calidad biológica del suelo, gracias a la marcada importancia que tienen en los procesos ecosistémicos y a su alta sensibilidad a los cambios en su hábitat natural (Decaëns et al. 2001; Lavelle et al. 2006; Bignell et al. 2008; Cabrera 2012; Junqueira et al. 2012). Particularmente, las termitas se pueden considerar como indicadoras útiles de los cambios generados por la intensificación de los suelos pues son uno de los grupos dominantes y más fácilmente observables dentro de las poblaciones de macroinvertebrados (Decäens et al. 2003). Estos insectos son esenciales para el mantenimiento de la estructura y funcionalidad del suelo, al mejorar su porosidad y aumentar la retención de agua gracias a su actividad microbiológica al interior del mismo (Alves et al. 2011). Además son altamente sensibles a las perturbaciones del medio o al cambio en el uso de la tierra
(Constantino y Schlemmermeyer 2000; Bandeira et al. 2003; Bandeira y Vasconcellos 2004; Ackerman et al. 2009).

Las sabanas tropicales de la Orinoquía Colombiana se han convertido en uno de los principales centros de desarrollo forestal en el país. En los últimos años se ha incrementado el establecimiento de plantaciones forestales, en terrenos previamente ocupados por sabanas naturales y pasturas introducidas, como una alternativa socioeconómicamente viable dentro de la región (Cortés-Pérez et al. 2005). Particularmente, Pinus caribaea Morelet (Pino caribe), es una de las especies más utilizadas para este propósito en la Meseta de San Pedro con cerca 1.525 ha sembradas dentro del núcleo forestal de la empresa Refocosta S.A. (Fernández Méndez et al. 2012).

Los cambios en la composición de la biodiversidad durante el ciclo de desarrollo de las plantaciones forestales son poco conocidos (De Camino y Budowski 1998). Evaluar y cuantificar los posibles efectos generados por el estableci-

\footnotetext{
${ }^{1}$ M. Sc. Estudiante. Universidad Distrital Francisco José de Caldas. Cr. 5 Este 15-85, Bogotá, D. C., Colombia, maira.beltran.diaz@gmail.com. ${ }^{2}$ Ph. D. Universidad Distrital Francisco José de Caldas. Cr. 5 Este 15-85, Bogotá, D. C., Colombia, opatriciap@udistrital.edu.co. Autor para correspondencia: M. Sc. Maira Alexandra Beltrán-Díaz. Estudiante. Universidad Distrital Francisco José de Caldas. Cr. 5 Este 15-85, Bogotá, D. C., Colombia, maira.beltran.diaz@) gmail.com.
} 
miento de monocultivos forestales es de gran importancia para determinar el grado de sostenibilidad ambiental de la actividad forestal, teniendo en cuenta que se pueden generar impactos negativos como pérdida de biodiversidad (Cruz 1988; González et al. 1996; Simberloff et al. 2009) o positivos como aumento de oferta de hábitat y de alimento para especies animales o vegetales (Rincón 2006). En este sentido, el uso de bioindicadores ha demostrado ser una herramienta útil y eficiente para visualizar el estado del ambiente, por medio de la presencia, ausencia o abundancia de especies que brindan información acerca de la calidad del ecosistema (Favila y Halffter 1997; Jamil 2001; Lavelle et al. 2006; Maleque et al. 2009). En este artículo se pretende estimar los cambios en la composición y estructura de la comunidad de termitas presentados luego del establecimiento, desarrollo y manejo de plantaciones forestales en suelos de pasturas y sabanas tropicales. Para esto se evaluó la composición de termitas en plantaciones de $P$. caribaea, en pasturas y en sabanas seminaturales, en un núcleo forestal de la Orinoquia ubicado en la Meseta de San Pedro en la Orinoquía colombiana.

\section{Materiales y métodos}

Área de estudio. La investigación se desarrolló en plantaciones de P. caribaea de 1.500 ha, de la empresa Refocosta localizadas en la meseta de San Pedro en el municipio de Villanueva (Casanare, Colombia) a $4^{\circ} 39.386^{\prime} \mathrm{N} 72^{\circ} 55.185^{\prime} \mathrm{O}$ y $358 \mathrm{msnm}$. La localidad presenta una temperatura media anual de $25,7^{\circ} \mathrm{C}$ y una precipitación anual de $2.911 \mathrm{~mm}$ con régimen monomodal, en el cual hay un periodo marcado de lluvias entre abril a noviembre y un periodo seco entre diciembre a marzo. La humedad relativa promedio es de 76 $\%$ siendo el periodo de mayo a septiembre el que presenta los mayores valores (hasta $85 \%$ ) y el comprendido entre diciembre y marzo el que registra los menores durante el año (Refocosta 2013).

El área del proyecto está constituida por los paisajes de sabanas inundables de la llanura eólica y las áreas rurales intervenidas no diferenciadas (Refocosta 2013). Particularmente, la Meseta de San Pedro corresponde a una terraza alta que está conformada de suelos arenosos a franco arenosos de naturaleza cuarzosa y de baja fertilidad pertenecientes a los órdenes entisol e inceptisol (Refocosta 2013).

Muestreo. Entre 2013 y 2014 se muestrearon dos lotes de $P$. caribaea por cada uno de los siguientes estadios: 1-2 años de edad, 6 -7 años de edad sin entresaca, 7-8 años de edad con entresaca y $19-23$ años de edad, siguiendo el protocolo estandarizado del transecto (Jones et al. 2005). Además se muestrearon dos lotes de pasturas y un lote de sabana seminatural. Se implementaron algunas modificaciones debido a la densidad de siembra de los lotes y la homogeneidad de los ecosistemas muestreados. De esta forma, la longitud y la distancia entre transectos estuvieron sujetas al interdistanciamiento de las plantaciones y tamaño de los lotes y el tiempo de muestreo estuvo condicionado por la baja complejidad de las áreas evaluadas. Se realizaron dos muestreos en cada lote, el primero en época húmeda (marzo) de 2013 y el segundo en época seca (noviembre) del 2013 y 2014.

Se instalaron tres transectos por lote, separados $50 \mathrm{~m}$ del borde del lote y entre sí por $20 \mathrm{~m}$ para un total de 33 transectos. Cada transecto tuvo una longitud de $50 \mathrm{~m}$, dividido en cinco subtransectos de $6 \mathrm{~m}$ por $3 \mathrm{~m}$ de ancho con una sepa- ración entre sí de $3 \mathrm{~m}$, para un total de ciento sesenta y cinco subtransectos.

Dentro de cada subtransecto se realizó un esfuerzo de muestreo de 40 min para un total de 3,5 horas por transecto. Se revisaron todos los montículos, ramas y secciones de tronco en contacto con el suelo y galerías presentes sobre los fustes de los árboles hasta $2 \mathrm{~m}$ de altura y se examinaron cuatro muestras de suelo de $12 \mathrm{~cm}$ x $12 \mathrm{~cm}$ por $10 \mathrm{~cm}$ de profundidad de los extremos de cada subtransecto para monitorear termitas del suelo. En pasturas y sabanas solo se realizó inspección de termitas de suelo y montículos, por la ausencia de madera sobre el suelo.

Preservación e identificación. Las termitas fueron recolectadas con pinzas finas y/o frascos aspiradores en etanol al 80 $\%$; posteriormente fueron limpiadas, marcadas y preservadas en viales de vidrio los cuales se encuentran depositados en la colección entomológica forestal adscrita al Laboratorio de Sanidad Forestal de la Universidad Distrital Francisco José de Caldas.

Cada muestra recolectada fue examinada con estereomicroscopios de alta resolución y con la ayuda de claves se determinó el nivel taxonómico más alto posible (Emerson y Banks 1957; Mathews 1977; Fontes 1982, 1986; Constantino 1995, 1999, 2001, 2002a, b; Cancello et al. 1996; Constantino y de Souza 1997; Cuezzo y Cancello 2009; Rocha et al. 2012; Oliveira 2013). Igualmente, se comparó con especímenes ya identificados de la colección entomológica de la Universidad Distrital. Por último, a cada muestra se le asignó el grupo trófico de acuerdo con la literatura (Constantino y Acioli 2006) y el sustrato de captura.

Variables fisionómicas, estructurales y de suelo. En cada transecto evaluado, se midieron parámetros fisionómicos de los individuos arbóreos como DAP (diámetro a la altura del pecho) utilizando cinta diamétrica y altura total con hipsómetro Haglof Vertx IV. Asimismo, se estimó la apertura del dosel como parámetro estructural de las plantaciones mediante el procesamiento de fotografías tomadas en transecto con una cámara reflex digital adaptada con un lente ojo de pescado de $5.6 \mathrm{~mm}$, instalada en un trípode, con el programa WinCAM V.2012a (Regent Instruments Canada Inc. 2012). Además, se tomó una muestra de suelo por cada lote, para obtener el contenido de humedad por el método tradicional de diferencia entre peso húmedo y seco; $\mathrm{pH}$ por el método de suspensión de suelo; contenido de nitrógeno por el método de Kjeldahl y contenido de carbono orgánico por el método de WalkleyBlack; los dos últimos análisis fueron solicitados al laboratorio de suelos de la Universidad Nacional de Colombia - sede Bogotá (Tabla 1).

Análisis de resultados. La eficiencia del muestreo se determinó a partir de curvas de acumulación de especies con ayuda del programa Estimate S 9.0 (Colwell 2009) y del análisis de completitud propuesto por Chao y Jost (2012), con ayuda del programa R-Project V. 3.3.2 (R Core Team 2013).

Para determinar la abundancia relativa de termitas se utilizó el número de encuentros por especie en cada subtransecto, de tal forma que si una especie estuvo presente en todos los subtransectos, tiene un valor de abundancia relativa de 15 por lote (Jones et al. 2005). La diversidad de termitas se analizó a través del índice de equidad de Shannon y Wiener $\left(\mathrm{H}^{\prime}\right)$, teniendo como base los datos de riqueza específica $(\mathrm{S})$ 


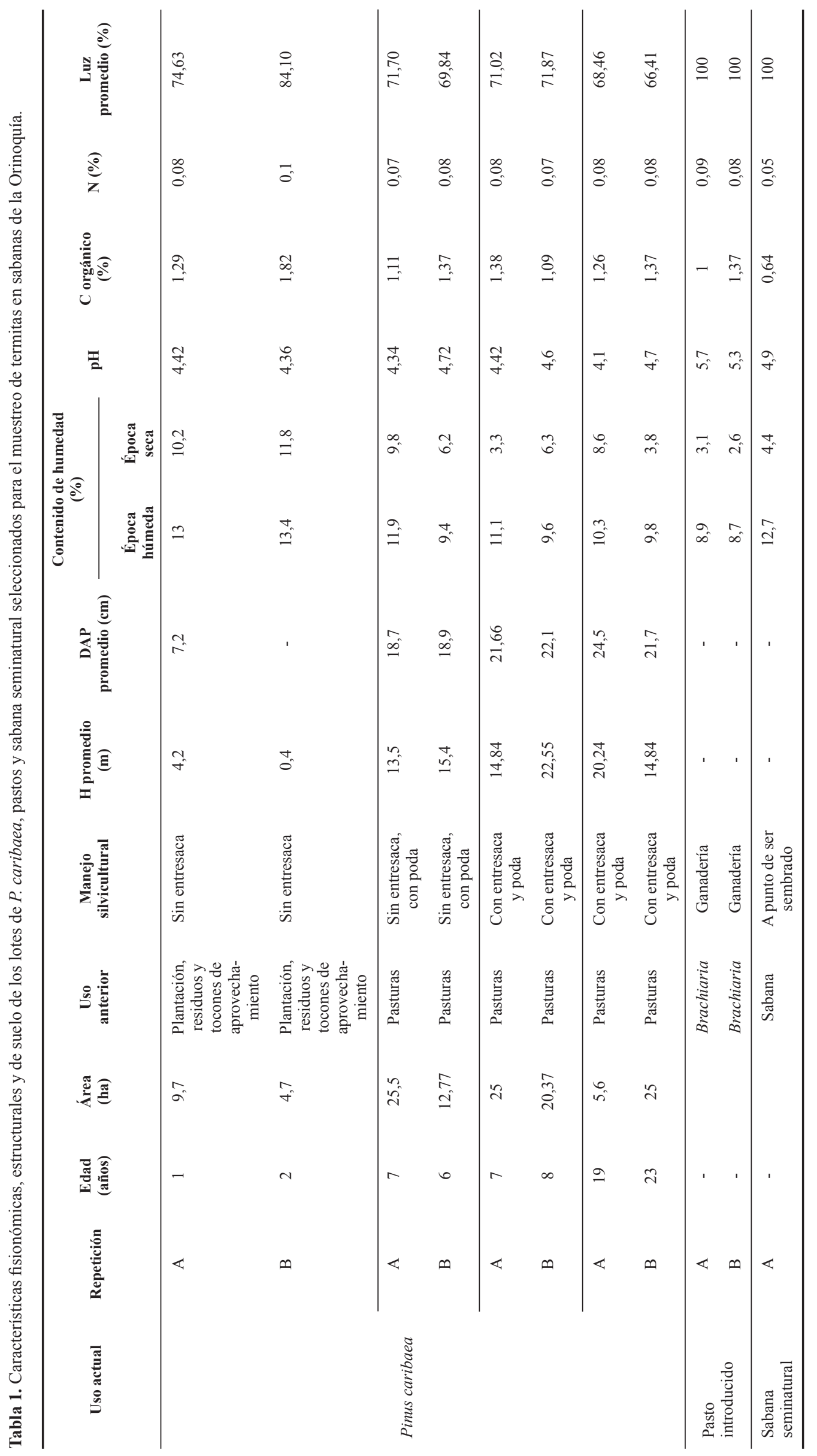




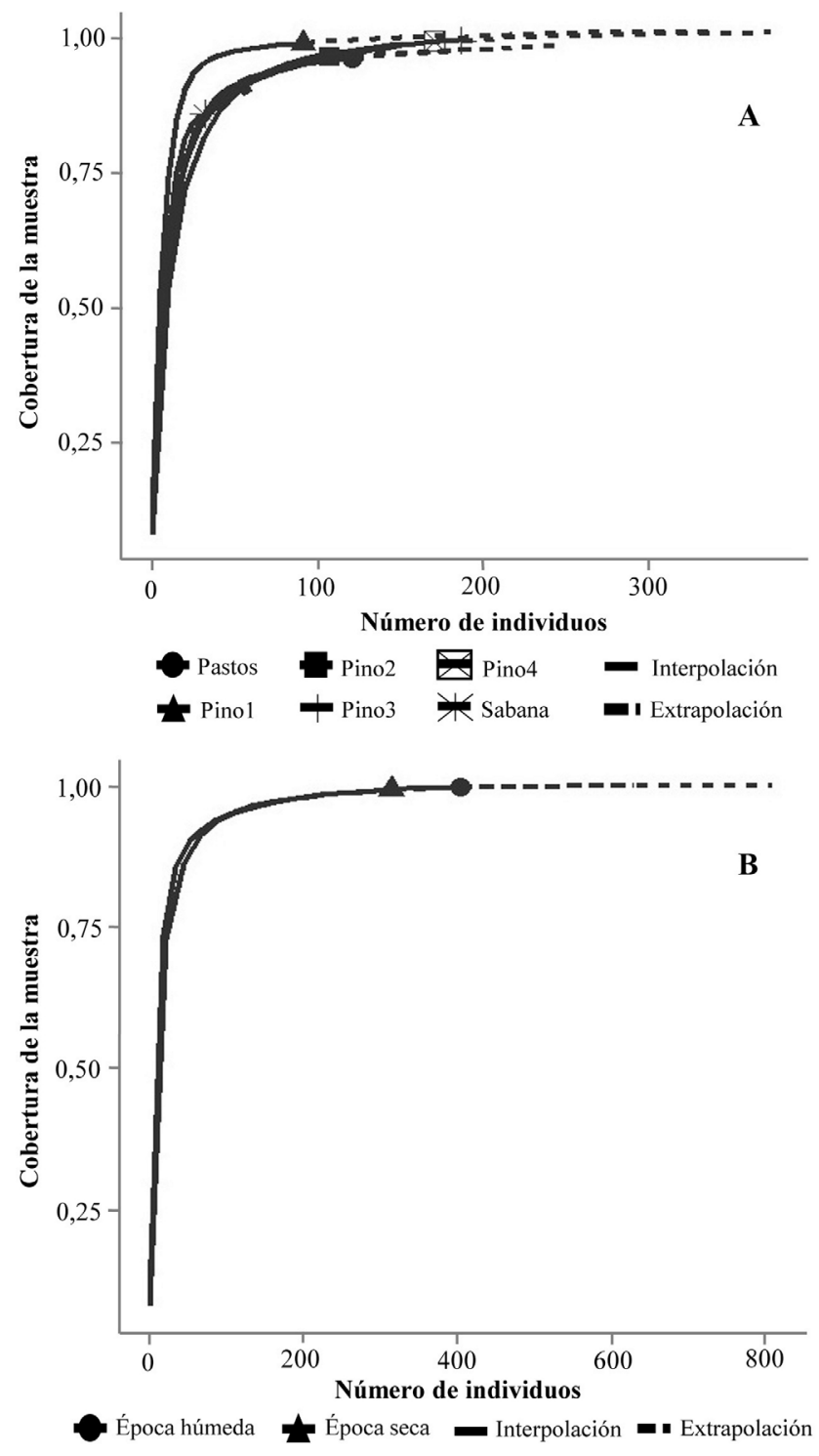

Figura 1. Curvas de eficiencia de muestreo de termitas en lotes de $P$. caribaea, pastos y sabanas seminaturales de la Orinoquía colombiana mediante análisis de completitud. A. Curva de cobertura de la muestra por uso del suelo. B. Curva de cobertura de la muestra por época de muestreo. Pino $1=\operatorname{Pc} 1 / 2$ años, Pino $2=P c 6 / 7$ años sin entresaca, Pino $3=\operatorname{Pc} 7 / 8$ años con poda y entresaca, Pino $4=\operatorname{Pc} 19 / 23$ años.

y abundancia relativa con ayuda del programa Estimate S 9.0 (Colwell 2009).

Una vez comprobada la normalidad de los datos con las pruebas de Shapiro-Wilk, se procedió a comparar la diversidad de termitas entre lotes y usos del suelo utilizando un análisis de varianza ANOVA de dos vías y, posteriormente, un test de Tukey y para la abundancia de termitas se empleó una prueba de proporciones con el valor $\mathrm{F}$ ajustado a la prueba de Holm.

Para establecer la relación entre la abundancia relativa de termitas como variable dependiente y las variables fisionómicas, estructurales y de suelo como variables independientes se utilizó un modelo de Poisson inflado de ceros, al ser la abundancia una variable respuesta de tipo categórica (conteo) con alta presencia de ceros. Por su parte, la correlación entre la diversidad de termitas y las variables explicativas se halló a partir de un análisis de covarianza, teniendo en cuenta que la diversidad es una variable respuesta de tipo cuantitativa. Además, se utilizó el método "stepwise" para realizar la selección final de variables explicativas y el criterio de información de Akaike y el criterio bayesiano de Schwarz, para determinar la veracidad de las variables seleccionadas. Todos los análisis estadísticos se realizaron utilizando el programa R-Project V. 3.3.2 (R Core Team 2013).

\section{Resultados}

Eficiencia del muestreo. En plantaciones de pino de 6 a 8 años con y sin entresaca y en pasturas se recolectaron cerca del $100 \%$ de las especies esperadas; mientras que en el resto de los lotes estudiados, la eficiencia del muestro estuvo alrededor del $80 \%$, por lo que se puede considerar que se obtuvo una buena representación del ensamblaje de termitas, lo que se evidenció en las curvas de cobertura de la muestra, las cuales se estabilizan con el número de individuos obtenidos (Fig. 1A). En cuanto a la época climática también se recolectaron cerca del $100 \%$ de las especies esperadas, dado que la curva se estabiliza con el número de individuos capturados (Fig. 1B).

Composición de termitas. Se recolectaron 28 especies distribuidas en dos familias (Termitidae y Rhinotermitidae), cinco subfamilias (Termitinae, Nasutitermitinae, Apicotermitinae, Rhinotermitinae, Heterotermitinae) y 19 géneros. El mayor número de especies (21) pertenece a la familia Termitidae, destacándose las subfamilias Nasutitermitinae y Apicoterminae con once y siete especies, respectivamente, del total recolectado (Tabla 2 ).

En plantaciones de pino de 6-7 años de edad sin entresaca se recolectó el mayor número de especies (24) variando entre época húmeda y seca de nueve a 18 especies. Luego se ubican las plantaciones de 7-8 años de edad con entresaca donde se registraron un total de 22 especies y una variación entre épocas de muestreo de diez a 17 especies. Por su parte, en los pastos fue donde se recolectó el menor número de especies, con un $31 \%$ de la riqueza total y una variación entre las épocas de estudio de siete a nueve especies (Tabla 2; Fig. 2A).

En todas las edades de plantación las subfamilias más abundantes en las dos épocas de muestreo fueron Apicotermitinae, representada por especies de Anoplotermes spp. y Heterotermitinae por Heterotermes tenuis y la menos abundante fue Syntermitinae la cual estuvo ausente en plantaciones de 1-2 años y pasturas. En pastos y sabanas predominó la subfamilia Apicotermitinae en las dos épocas de muestreo (Tabla 2; Fig. 2B).

Composición de grupos tróficos. Se recolectaron termitas pertenecientes a cuatro grupos tróficos como son xilófagas (alimentación a base de madera no descompuesta), humívoras (alimentación a base de humus o partículas minerales de la mezcla suelo-madera), intermedias (alimentación a base de madera descompuesta y humus) y filófagas (alimentación a base de hojarasca).

El grupo humívoro presentó el mayor número de especies $(38 \%)$ seguidos por termitas de hábitos intermedios $(27 \%)$, xilófagas $(24 \%)$ y filófagas $(10 \%)$. En plantaciones de pino de todas las edades la diversidad de grupos tróficos estuvo representada principalmente por especies humívoras y xilófagas y en pasturas y sabanas por especies humívoras en las dos épocas de muestro. (Tabla 2; Figs. 2C y 2D). 


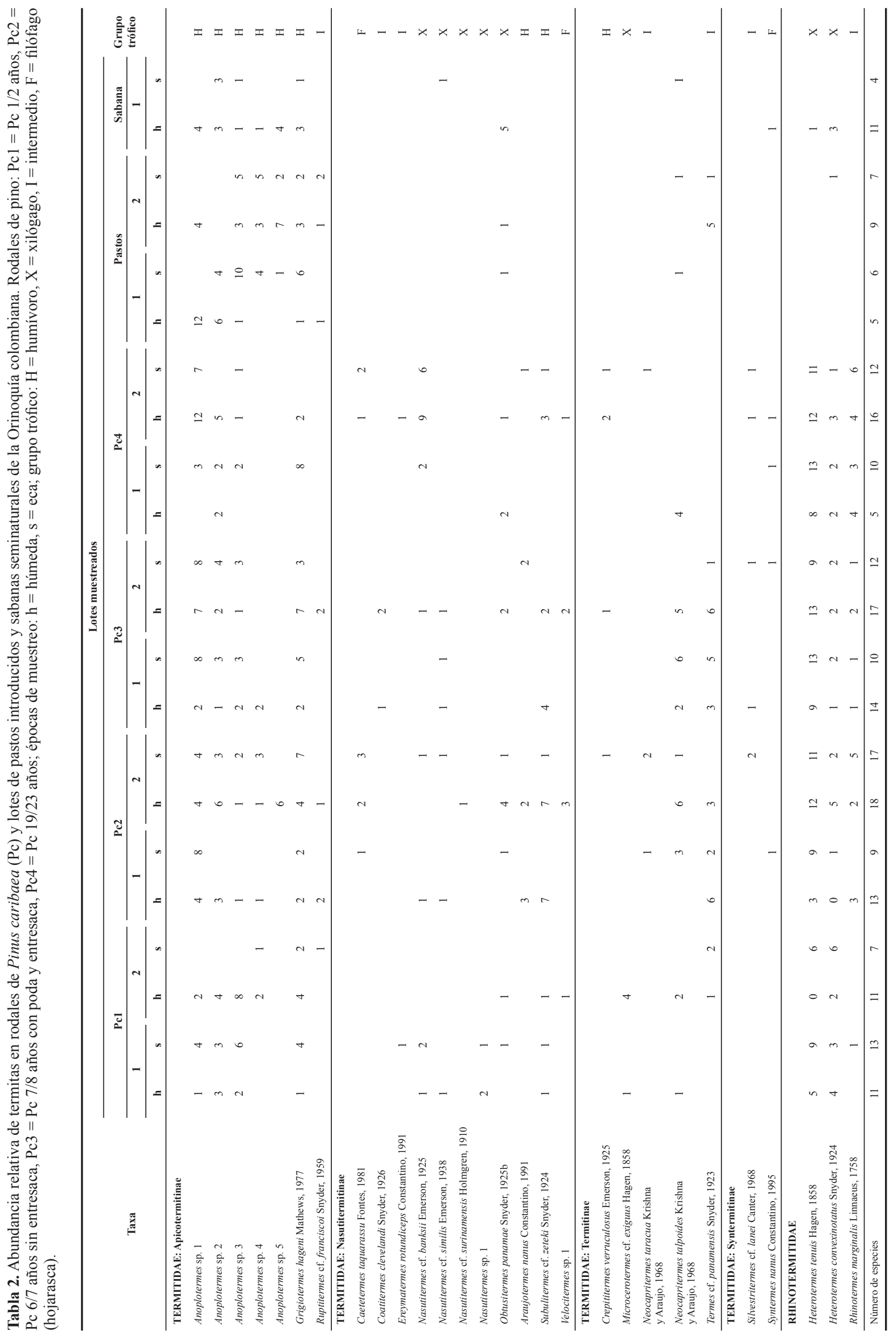



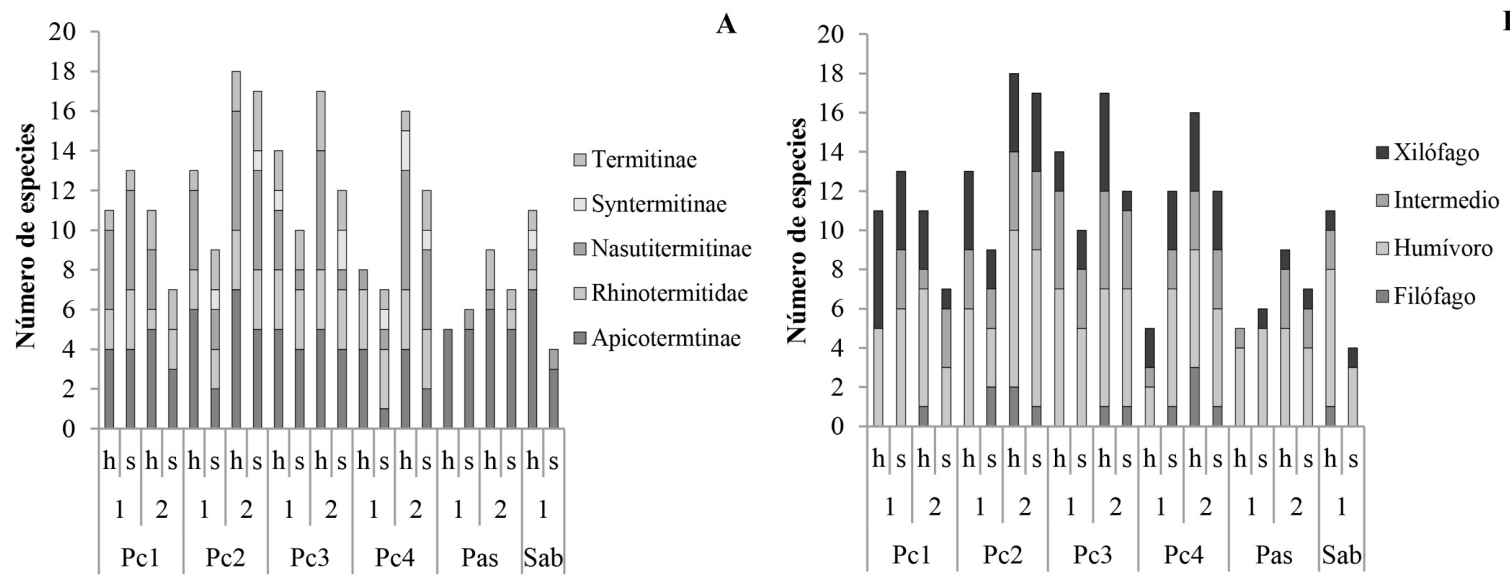

B
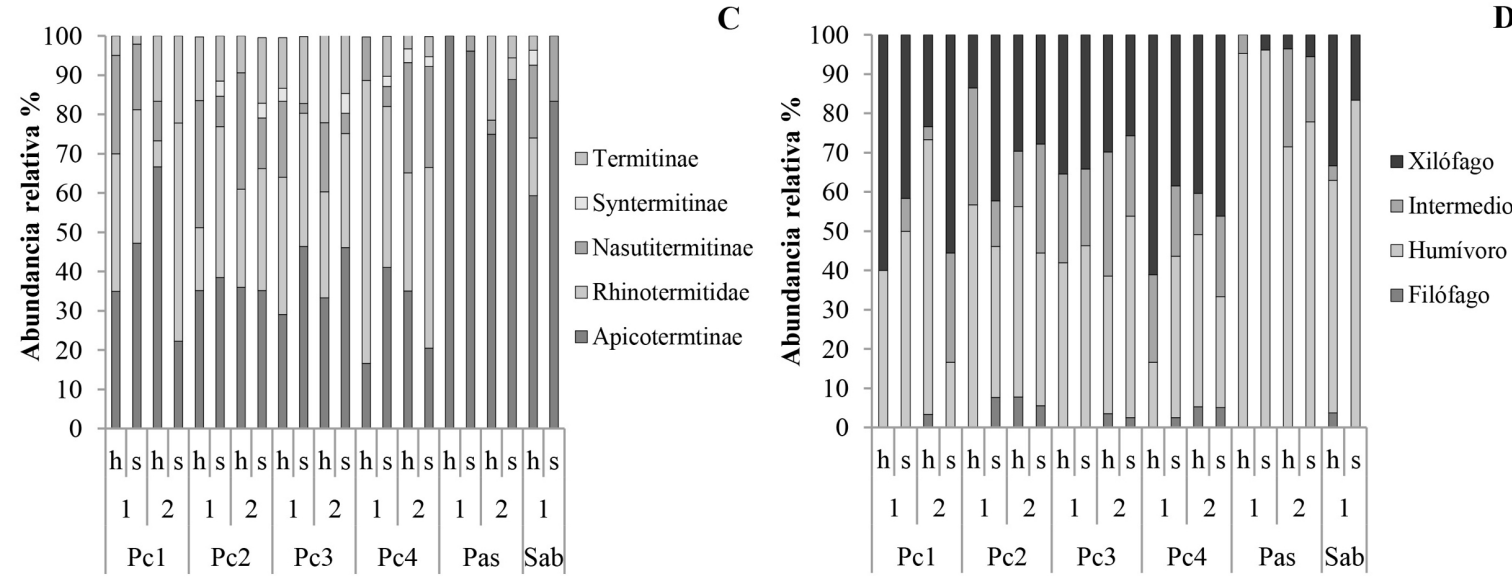

Figura 2. Riqueza y abundancia de termitas en lotes de P. caribaea (Pc), pastos (Pas) y sabanas seminaturales (sab) de la Orinoquía colombiana. A. Número de especies por subfamilia. B. Abundancia relativa por subfamilia. C. Número de especies por grupo trófico. D. Abundancia relativa por grupo trófico. $\mathrm{Pc} 1=\mathrm{Pc}$ de $1 / 2$ años, $\mathrm{Pc} 2=\mathrm{Pc} 6 / 7$ años sin entresaca, $\mathrm{Pc} 3=\mathrm{Pc} 7 / 8$ años con poda y entresaca, Pc4 = Pc 19 y 23 años de edad. h = Época húmeda, $\mathrm{s}=$ Época seca.

Diversidad de termitas. En los lotes de pino se encontraron diferencias estadísticamente significativas en la diversidad de termitas comparada con las pasturas y sabanas seminaturales $\left(\mathrm{S}: \mathrm{P}=1,33 \mathrm{e}^{-05}, \mathrm{H}^{\prime}: \mathrm{P}=0,0001991 ; \mathrm{gl}=10\right)$ y la edad de la plantación ( $\mathrm{S}$ : $\mathrm{P}=0,0004554, \mathrm{H}^{\prime}: \mathrm{P}=0,01237$; $\mathrm{gl}=$ 7). Entre sabanas y pastos la estructura de la termitofauna fue similar ( $\mathrm{S}: \mathrm{P}=0,2481 ; \mathrm{H}^{\prime}: \mathrm{P}=0,1118, \mathrm{gl}=2$ ) (Tabla 3). Referente a las épocas de muestreo no se encontraron diferencias significativas $\left(\mathrm{S}: \mathrm{P}=0,1015, \mathrm{H}^{\prime}: \mathrm{P}=0,05420\right.$; $\mathrm{gl}=7)($ Tabla 3$)$.

Tabla 3. Valores de $\mathrm{P}$ obtenidos en el análisis de varianza y en la prueba de proporciones para comparar riqueza y abundancia de especies con usos del suelo, edades de plantaciones y época de muestreo.

\begin{tabular}{|c|c|c|c|}
\hline \multirow{2}{*}{ Variables } & \multicolumn{2}{|c|}{$\begin{array}{c}\text { Diversidad } \\
\text { (Análisis de varianza) }\end{array}$} & \multirow{2}{*}{$\begin{array}{c}\text { Abundancia } \\
\text { (Prueba de } \\
\text { proporciones) }\end{array}$} \\
\hline & Específica & $\begin{array}{l}\text { Shannon y } \\
\text { Wiener }\end{array}$ & \\
\hline Uso del suelo & $1,33 \mathrm{e}^{-05 *}$ & $1,99 \mathrm{e}^{-04 *}$ & $3,5 \mathrm{e}^{-07 *}$ \\
\hline Edad de plantación & $4,55 \mathrm{e}^{-04 *}$ & $0,01237 *$ & $7,6 \mathrm{e}^{-06 *}$ \\
\hline Pastos y sabanas & 0,2811 & 0,1118 & 0,9112 \\
\hline Época & 0,1015 & 0,0542 & 0,2521 \\
\hline
\end{tabular}

Nivel de confianza de $95 \%$ * Variables que presentaron significancia estadística.
Las termitas xilófagas fueron más diversas en plantaciones de pino con respecto a las pasturas y las sabanas seminaturales $\left(\mathrm{S}: \mathrm{P}=4,17 \mathrm{e}^{-08}, \mathrm{H}^{\prime} \mathrm{P}=0,00015 ; \mathrm{gl}=10\right)$ y se encontró mayor número en época húmeda $\left(\mathrm{S}: \mathrm{P}=0,0117, \mathrm{H}^{\prime} \mathrm{P}=\right.$ $0,0017 ; \mathrm{gl}=10)$. Las termitas de hábitos intermedios fueron más diversas en plantaciones de edad media y adulta en comparación con las pasturas y las plantaciones recién establecidas ( $\left.\mathrm{S}: \mathrm{P}=0,02306, \mathrm{H}^{\prime} \mathrm{P}=0,01023 ; \mathrm{gl}=10\right)$, sin diferencias significativas para la época de muestreo $\left(\mathrm{S}: \mathrm{P}=0,81039, \mathrm{H}^{\prime}\right.$ $\mathrm{P}=0,2456 ; \mathrm{gl}=10)$. No se encontraron diferencias significativas en la diversidad de termitas humívoras con respecto a los usos del suelo y época de muestreo ( $\mathrm{S}: \mathrm{P}=0,1256, \mathrm{H}^{\prime} \mathrm{P}=$ $0,2448 ; \mathrm{gl}=10)($ Tabla 4).

Abundancia de termitas. En plantaciones de pino, la abundancia de termitas fue significativamente mayor en el pino de 6 años sin entresaca (185 encuentros) comparada con la del pino 1 año (105 encuentros) $\left(\mathrm{P}=7,6 \mathrm{e}^{-06}, \mathrm{gl}=3\right)$, sin diferencias importantes en las épocas de muestreo $(\mathrm{P}=0,25 ; \mathrm{gl}=1)$ (Tabla 3). Las especies más abundantes fueron Heterotermes tenuis y Anoplotermes sp. 1 con 23 y $12 \%$ de los encuentros registrados durante el muestreo $\left(H\right.$. tenuis $\left(\mathrm{P}=<2 \mathrm{e}^{-16} ; \mathrm{gl}=\right.$ 7), A. sp. $\left.1\left(\mathrm{P}=1,2 \mathrm{e}^{-15} ; \mathrm{gl}=7\right)\right)$. Los grupos tróficos con mayor representación dentro de las plantaciones fue el xilófago y el humívoro con un $40 \%$ más de encuentros que el grupo 
Tabla 4. Valores de $\mathrm{P}$ obtenidos en el análisis de varianza y en la prueba de proporciones para comparar riqueza y abundancia de grupos tróficos con usos del suelo y época de muestreo.

\begin{tabular}{|c|c|c|c|c|}
\hline \multirow{2}{*}{\multicolumn{2}{|c|}{ Variables }} & \multicolumn{2}{|c|}{$\begin{array}{c}\text { Diversidad } \\
\text { Análisis de varianza }\end{array}$} & \multirow{2}{*}{$\begin{array}{c}\text { Abundancia } \\
\text { (Prueba de } \\
\text { proporciones) }\end{array}$} \\
\hline & & Específica & $\begin{array}{l}\text { Shannon y } \\
\text { Wiener }\end{array}$ & \\
\hline \multirow{3}{*}{ Uso del suelo } & Xilófago & $4,17 \mathrm{e}^{-08 *}$ & $0,00015 *$ & $5,1 \mathrm{e}^{-13 *}$ \\
\hline & Intermedios & $0,02306 *$ & $0,01023 *$ & 0,1571 \\
\hline & Humívoro & 0,1256 & 0,2448 & $7,6 \mathrm{e}^{-07 *}$ \\
\hline \multirow{3}{*}{$\begin{array}{l}\text { Época de } \\
\text { muestreo }\end{array}$} & Xilófago & $0,0117 *$ & $0,0017^{*}$ & 0,2432 \\
\hline & Intermedios & 0,8104 & 0,2451 & 0,1489 \\
\hline & Humívoro & 0,1161 & 0,1892 & $0,0081 *$ \\
\hline
\end{tabular}

Nivel de confianza de $95 \%$. * Variables que presentaron significancia estadística.

filófago (xilófago con $\mathrm{P}=5,1 \mathrm{e}^{-13}$; humívoro con $\mathrm{P}=7,6 \mathrm{e}^{-07}$; $\mathrm{gl}=3$ ) (Tablas 3 y 4).

Entre pastos y sabanas el encuentro de termitas fue simi$\operatorname{lar}(\mathrm{P}=0,91, \mathrm{gl}=5)$. En pastos la especie más recolectada fue Anoplotermes sp. $3(\mathrm{P}=0,010, \mathrm{gl}=3)$ y el grupo trófico con mayor frecuencia de encuentros fue humívoro $\left(\mathrm{P}=1,7 \mathrm{e}^{-08} ; \mathrm{gl}\right.$ $=3)$, sin diferencias en época de muestreo $(\mathrm{P}=0,91 ; \mathrm{gl}=3)$. En sabana seminatural se registró mayor número de encuentros en época húmeda $(\mathrm{P}=0,0081 ; \mathrm{gl}=3)$, ninguna de las especies fue predominante y el grupo trófico humívoro fue el más frecuente $(\mathrm{P}=0,00025, \mathrm{gl}=3)$ (Tablas 3 y 4$)$.

Diversidad y abundancia de termitas según las características del suelo y la plantación. De las diez variables incluidas en el muestreo, la edad de la plantación, el diámetro a la altura del pecho (DAP), el porcentaje de luz, el contenido de carbono orgánico del suelo y el contenido de nitrógeno total, contribuyeron a explicar la diversidad de termitas (Tabla 5).

Tabla 5. Valores de $\mathrm{P}$ obtenidos en el análisis de covarianza y en el análisis de Poisson inflado de ceros para la relación entre riqueza y abundancia de especies y variables ambientales evaluadas.

\begin{tabular}{lccccc}
\hline \multicolumn{1}{c}{ Variables } & \multicolumn{2}{c}{$\begin{array}{c}\text { Riqueza } \\
\text { (Análisis de covarianza) }\end{array}$} & & \multicolumn{2}{c}{$\begin{array}{c}\text { Abundancia } \\
\text { (Análisis de Poisson inflado de ceros) }\end{array}$} \\
\cline { 2 - 3 } \cline { 5 - 6 } & Específica & Shanon & & Anoplotermes sp. 1 & Heterotermes tenuis \\
\hline Uso del suelo-edad & $0,00020 * *$ & $0,00027 * *$ & & 0,12150 & 0,06785 \\
Epoca & 0,67770 & 0,74760 & & 0,73420 & 0,30030 \\
DAP & $0,00028 *$ & $0,00057 *$ & & 0,96420 & $0,02103 *$ \\
Altura & $0,06400 *$ & $0,10800 *$ & & 0,98130 & $0,01102 *$ \\
Porcentaje de luz & $0,00039 *$ & $0,00143 *$ & & 0,48480 & $0,01227 *$ \\
Contenido humedad suelo & 0,16450 & 0,11380 & & 0,25890 & 0,05937 \\
pH & 0,33020 & 0,14030 & & $0,02898 *$ & 0,88700 \\
Carbono orgánico & $0,00075 *$ & $0,00070 *$ & & 0,38870 & 0,44140 \\
Nitrógeno total & $0,04176 *$ & $0,00221 *$ & & 0,27470 & 0,60400 \\
Acumulación de hojarasca & 0,64600 & 0,13030 & & 0,87300 & 0,58180 \\
\hline
\end{tabular}

Nivel de confianza de $95 \%$. Variables respuesta que se relacionaron con la variable dependiente.

** Variable excluida del análisis por presentar multicolinealidad.

Tabla 6. Valores de ajuste obtenidos para las variables ambientales relacionadas con la riqueza y abundancia de especies de termitas según los criterios de información de Akaike y el bayesiano de Schwartz.

\begin{tabular}{lccccc}
\hline \multirow{2}{*}{$\begin{array}{c}\text { Criterio de ajuste } \\
\text { de variables }\end{array}$} & $\begin{array}{c}\text { Rodelo 1: variables } \\
\text { que obtuvieron } \\
\text { relación }\end{array}$ & $\begin{array}{c}\text { Modelo 2: variables } \\
\text { seleccionadas }\end{array}$ & & $\begin{array}{c}\text { Modelo 1: variables } \\
\text { que obtuvieron } \\
\text { relación }\end{array}$ & $\begin{array}{c}\text { Modelo 2: variables } \\
\text { seleccionadas }\end{array}$ \\
\hline Akaike & 97,694 & & & 206,023 & $202,635^{*}$ \\
Schwartz & 110,832 & $102,6767 *$ & & N/A ** & N/A ** \\
\hline
\end{tabular}

* Los valores más bajos indican certeza en la selección de las variables. ** El criterio de Schwart no aplica para el análisis de Poisson inflado de ceros. 
Sin embargo, la variable tipo de uso del suelo - edad presentó multicolinealidad con las demás variables explicativas por lo que fue eliminada del análisis de covarianza. La selección de variables permitió determinar que el nitrógeno total del suelo y el porcentaje de luz fueron las dos variables que en conjunto explicaron mejor la diversidad de termitas encontrada en el muestreo $\left(\mathrm{P}_{\text {nitrógeno total }}=0,0008971, \mathrm{p}_{\text {porcentaje de luz }}=0,0005852\right.$; $\mathrm{gl}=1)$ (Tabla 6).

En términos de abundancia, se tomaron como referencia las dos especies que fueron significativamente más abundantes dentro del muestreo (Anoplotermes sp. 1 y Heterotermes tenuis), para determinar su relación con las variables ambientales medidas. Se encontró que Anoplotermes. sp. 1 se relacionó únicamente con el $\mathrm{pH}$ del suelo $(\mathrm{P}=0,02898, \mathrm{gl}=1)$, mientras $H$. tenuis se relacionó con el porcentaje de luz, la altura de los árboles y el diámetro a la altura del pecho $\left(\mathrm{P}_{\text {por- }}\right.$ centaje de luz $=0.01270, \mathrm{pH}=0,01102, \mathrm{pDAP}=0,02103 ; \mathrm{gl}=2$ ) (Tabla 5). Una vez seleccionadas las variables se determinó que la abundancia de $H$. tenuis se explicó en mayor medida por el porcentaje de luz, mostrando una correlación negativa con esta variable $\left(\mathrm{P}_{\text {porcentaje de luz }}=0,0254 ; \mathrm{gl}=1\right)($ Tabla 6$)$.

\section{Discusión}

En el presente estudio no se registraron especies de la familia Kalotermitidae, a pesar de que ser la segunda familia más diversa en el Neotrópico (Pearce y Waite 1994). Esto puede deberse a que estas termitas se desarrollan específicamente en madera seca que no necesariamente debe estar en contacto con el suelo (Krishna 1961), por lo que las condiciones microclimáticas generadas dentro de las plantaciones de pino hacen que las piezas de madera no brinden las condiciones necesarias para el establecimiento de especies de termitas de esta familia.

Las plantaciones de pino de todas las edades estuvieron dominadas por especies humívoras $(43,4 \%$ promedio para las cuatro edades) seguida de especies xilófagas $(21,9$ $\%$ promedio para las cuatro edades) en las dos épocas de muestreo. Esta misma tendencia se ha podido observar en plantaciones homogéneas de Eucalyptus sp. y Hevea brasiliensis en donde las termitas del suelo son significativamente abundantes y diversas en plantaciones de todas las edades y hay un aumento en la abundancia de especies xilófagas en plantaciones de mayor edad (Junqueira y Diehl 2009; Pinzón et al. 2012).

Heterotermes tenuis estuvo presente en todas las edades de plantación en las dos épocas de muestreo, siendo muy abundante en las plantaciones maduras de 7 años con poda y 19-23 años de edad. Es importante tener en cuenta que las termitas xilófagas son resistentes a la alteración del medio y son las primeras en recolonizar el hábitat después de su modificación (Constantino y Acioli 2006; Eggleton et al. 1995; Eggleton y Tayasu 2001), además, la distribución de estas especies de termitas va de acuerdo a la disponibilidad de recursos presentes en el ambiente (Houston et al. 2014), por lo que el establecimiento de las plantaciones y la acumulación de piezas madera sobre el suelo en las plantaciones adultas puede aumentar la presencia de especies de este grupo trófico.

Así mismo, se ha registrado que ecosistemas con alta concentración de hojarasca en el suelo, favorecen la densidad de algunas especies xilófagas como termitas del género Heterotermes sp. (Goncalves et al. 2004; Polatto y Alves-Junior
2009), de tal forma que la densa capa de acículas sobre el suelo de las plantaciones permite que la actividad de forrajeo de estas especies de termitas sea constante. Los resultados encontrados en este estudio coinciden con lo observado en plantaciones comerciales de eucalipto en Brasil en donde se registra a $H$. tenuis como una especie muy abundante en rodales adultos (Junqueira y Diehl 2009) y en plantaciones de caucho de la Orinoquía colombiana donde $H$. convexinotatus es la especie xilófaga predominante, en especial en plantaciones adultas mayores a 10 años de edad (Pinzón et al. 2012).

En ecosistemas tropicales se registra una mayor abundancia de termitas en época húmeda (Basu et al. 1996), sin embargo, la fluctuación en la abundancia de termitas humívoras en particular de la especie Anoplotermes spp. entre régimen climático en todos los usos del suelo y de la especie $H$. tenuis en todas las edades de plantación, no fue muy contrastante, debido probablemente a la diversidad de dieta que exploran las termitas subterráneas (Abensperg-Traun 1991); y a que la profundidad del forrajeo depende en gran medida de la humedad relativa y la temperatura del suelo (Cornelius y Osbrink 2011). El dosel cerrado mantiene la humedad del suelo y de las piezas de madera, lo que puede favorecer que las termitas forrajeen y permanezcan en las capas más superficiales del suelo y la hojarasca. Por el contrario, durante la época seca y durante el día para protegerse de desecación se profundizan en el suelo (Nobre et al. 2009).

Durante la época seca las termitas se desplazan a horizontes profundos del suelo para protegerse de la exposición solar directa, debido al aumento de la temperatura y la disminución de la humedad relativa y la humedad del suelo (Nobre et al. 2009). Dada la característica de que las termitas conforman nidos y estos no son móviles (Noirot y Darlington 2000), la menor diversidad durante la época seca en nuestro estudio, podría explicarse por una menor actividad de las termitas en galerías y capas superficiales del suelo que es a donde está enfocado este tipo de muestreo más que a una disminución real del número de especies.

La representatividad del grupo humívoro encontrada en pasturas y sabanas seminaturales en las dos épocas de muestreo puede deberse a que en ecosistemas neotropicales tiende a haber mayor diversidad de especies de este grupo gracias a la especialización de su nicho por debajo del suelo (Bourguignon et al. 2009). Además se ha podido comprobar que en sabanas naturales y pasturas hay predominancia de especies humívoras, siendo las sabanas naturales las de mayor diversidad y abundancia (Basu et al. 1996; Benito et al. 2004; Alves et al. 2011; Cabrera 2012).

Si bien la abundancia de termitas no fue diferente entre pastos introducidos y sabanas, se observa que en sabana hay mayor riqueza específica (11 especies) y aparecen otros grupos tróficos como el filófago (Syntermes cf. nanus), posiblemente porque en este último hábitat hay presencia de especies vegetales herbáceas y gramíneas que mantienen una cobertura vegetal permanente sobre el suelo y aumentan la oferta de alimento. Esto reafirma a su vez cómo las termitas son sensibles a la perturbación del medio mostrando una alteración notoria en su composición con el cambio de uso del suelo, debido a las modificaciones microclimáticas y de oferta de alimento (Eggleton et al. 1995; Lavelle et al. 1997; Bandeira et al. 2003; Bandeira y Vasconcellos 2004).

Las termitas del género Anoplotermes son poco selectivas en el origen de la materia orgánica de la que se alimentan (Bourguignon et al. 2009), lo que puede explicar su presencia 
en todos los lotes de muestreo y su abundancia significativa en pasturas artificiales; de tal manera que la acumulación de estiércol de semovientes producto de la actividad agropecuaria de la zona puede influir en la presencia de especies de Apicotermitinae en este uso del suelo.

Es bien sabido que las termitas exploran diversos recursos alimenticios desde madera viva o muerta hasta gramíneas, hojarasca, hongos, nidos y excremento de animales; además el estiércol es rico en nitrógeno y las termitas de suelo son descomponedores primarios de este tipo de recurso en especial en hábitats abiertos (Lima y Costa-Leonardo 2007), lo que puede explicar la relación hallada entre la diversidad de termitas y el contenido de nitrógeno total en el suelo (Tabla 6).

La correlación negativa encontrada entre la diversidad y abundancia de termitas con el porcentaje de luz, puede deberse a que en la medida que las plantaciones de pino maduran, van cerrando su dosel y de esta forma las condiciones de humedad y temperatura en el ambiente no presentan fluctuaciones tan abruptas como en ecosistemas sin cobertura vegetal. Esta misma tendencia se observó en plantaciones comerciales de $H$. brasiliensis de los llanos orientales de Colombia donde hubo predominancia de especies humívoras y xilófagas en plantaciones adultas con el dosel más cerrado (Pinzón et al. 2012) y sistemas agrosilviculturales de Terminalia ivorensis en el sur de Camerún, en donde la abundancia de termitas de suelo fue mayor en plantaciones adultas con alta densidad de copa (Dibog et al. 1999).

Es importante mencionar que no se observó evidencia de daño de termitas sobre árboles vivos y sanos; de igual forma P. caribaea se reporta como una especie resistente al ataque de termitas debido a su alto contenido de resina (Francis y Forest Service 1992; Arguedas-Gamboa 2012). Esto sugiere que la presencia de termitas xilófagas e intermedias dentro de las plantaciones, puede estar contribuyendo en la descomposición de residuos de madera sobre el suelo y que la alta abundancia de termitas humívoras puede estar aportando en la descomposición de la materia orgánica y en el mejoramiento de la fertilidad del suelo.

En regiones tropicales como Jamaica, África y Suramérica se ha observado que el establecimiento de plantaciones de especies coníferas como $P$. caribaeae disminuye la abundancia o diversidad de algunos grupos de insectos como hormigas y escarabajos (Cruz 1988; González et al. 1996; Simberloff et al. 2009), especialmente cuando esta actividad se realiza alterando los ecosistemas naturales. Sin embargo, en la presente investigación, la diversidad y abundancia de termitas en las plantaciones de pino aumentó en comparación con la de las pasturas y sabanas seminaturales; posiblemente debido al mejoramiento de las condiciones microclimáticas y de oferta de alimento. Es importante tener en cuenta que dentro de la zona donde se sembraron las plantaciones de pino evaluadas, se conservaron las áreas de bosque natural, principalmente las que rodean las fuentes hídricas y que se conocen como bosques de galería y dentro de este núcleo forestal comercial se mantienen sin intervención, lo que sugiere que las plantaciones están actuando como corredores biológicos para las especies de termitas propias de estos bosques.

En conclusión, el establecimiento de plantaciones de $P$. caribaeae en terrenos previamente ocupados por pasturas y sabanas naturales crea condiciones favorables para la llegada y establecimiento de termitas xilófagas, humívoras, intermedias y filófagas que pueden provenir de ecosistemas aledaños, gracias al aumento de la oferta alimenticia y a la provisión de condiciones adecuadas para la actividad de forrajeo de las especies; de tal manera que las termitas entran a jugar un papel importante en la dinámica natural de las plantaciones al contribuir en la descomposición del material celulósico acumulado sobre el suelo y aportando en la fertilidad del mismo.

\section{Agradecimientos}

Las autoras agradecen al Centro de Investigación y Desarrollo Científico de la Universidad Distrital "Francisco José de Caldas" por el apoyo financiero dentro del proyecto No. 55 de 2012. A la empresa Refocosta S. A. y a los Ingenieros Mónica Sarmiento y Guido Gasca por el apoyo logístico. A Natalia Arias, Loren Baquero, Jeffer Vega y Daniel Castro por su apoyo en campo. A los profesores Andrés Ortiz y Jaime Pinzón y a Walter García por la asesoría estadística, a la profesora María Alejandra Jaramillo y a revisores anónimos que hicieron importantes contribuciones a versiones anteriores del manuscrito.

\section{Literatura citada}

ABENSPERG-TRAUN, M. 1991. Seasonal changes in activity of subterranean termite species (Isoptera) in Western Australian wheat belt habitats. Australian Journal of Ecology 16 (3): 331336.

ACKERMAN, I. L.; CONSTANTINO, R.; GAUCH JR, H. G.; LEHMANN, J.; RIHA, S. J.; FERNANDES, E. 2009. Termite (Insecta: Isoptera) species composition in a primary rain forest and agroforests in Central Amazonia. Biotropica 41 (2): 226-233.

ALVES, W. DE F.; MOTA, A. S.; LIMA, R. DE BELLEZONI R.; VASCONCELLOS, A. 2011. Termites as bioindicators of habitat quality in the caatinga, Brazil: Is there agreement between structural habitat variables and the sampled assemblages?. Neotropical Entomology 40 (1): 39-46.

ARGUEDAS-GAMBOA, M. 2012. Plagas y enfermedades forestales en Costa Rica. Revista Forestal Mesoamericana Kurú 4 (11-12): 1-69.

BANDEIRA, A. G.; VASCONCELLOS, A. 2004. Efeitos de perturbações antrópicas sobre as populações de cupins (Isoptera) do Brejo dos Cavalos, Pernambuco. pp. 145-152. En: Porto, K; Cabral, J. y Taberelli, M. (Eds.). Brejos de Altitude em Pernambuco e Paraíba: história natural, ecologia e conservação. Brasília: Ministério do Meio Ambiente. 324 p.

BANDEIRA, A. G.; VASCONCELLOS, A.; SILVA, M. P.; CONSTANTINO, R. 2003. Effects of habitat disturbance on the termite fauna in a 117 highland humid forest in the Caatinga domain, Brazil. Sociobiology 42 (1): 117-127.

BASU, P.; BLANCHART, E.; LEPAGE, M. 1996. Termite (Isoptera) community in the Western Ghats, South India: influence of anthropogenic disturbance of natural vegetation. European Journal of Soil Biology 32 (3): 113-121.

BENITO, N. P.; BROSSARD, M.; PASINI, A.; GUIMARÃES, M.; BOBILLIER, B. 2004. Transformations of soil macroinvertebrate populations after native vegetation conversion to pasture cultivation (Brazilian Cerrado). European Journal of Soil Biology 40 (3): 147-154.

BIGNELL, D. E.; CONSTANTINO, R.; CZUDI, C. 2008. Macrofauna. pp. 43-75. En: Moreira, F; Huising, E.; Bignell, D. (Eds.). A handbook of tropical soil biology: sampling and characterization of below-ground biodiversity. Earthscan, Londres. 248 p.

BOURGUIGNON, T.; ŠOBOTNÍK, J.; LEPOINT, G.; MARTIN, J.-M.; ROISIN, Y. 2009. Niche differentiation among neotropical soldierless soil-feeding termites revealed by stable isotope ratios. Soil Biology and Biochemistry 41 (10): 2038-2043. 
CABRERA, G. 2012. La macrofauna edáfica como indicador biológico del estado de conservación/perturbación del suelo. Resultados obtenidos en Cuba. Pastos y Forrajes 35 (4): 346-363.

CANCELLO, E.; BRANDÁO, D.; AMARANTE S. T. P. 1996. Two new Angularitermes species (Isoptera, Termitidae, Nasutitermitnae) from Brazil with a discussion of the cephalic microsculpture of the soldier. Sociobiology 27 (3): 277- 287.

CHAO, A; JOST, L. 2012. Coverage-based rarefaction and extrapolation: standardizing samples by completeness rather than size. Ecology 93 (12): 2533-2547.

COLWELL, R. K. 2009. Estimate S: Statistical estimation of species richness and shared species from samples. Versión 9.0. Disponible en: http://viceroy.eeb.uconn.edu/EstimateS/.[Fecha revisión: 22 mayo 2015].

CONSTANTINO, R. 1995. Revision of Neotropical termite genus Syntermes Holmgren (Isoptera: Termitidae). The University of Kansas. Science Bulletin 55: 455-518.

CONSTANTINO, R. 1999. Chave ilustrada para identificacáo dos géneros de cupins (Insecta: Isoptera) que ocorrem no Brasil. Papeis Avulsos de Zoología 40 (25): 387-448.

CONSTANTINO, R. 2001. Key to soldiers of South American Heterotermes with a new species from Brazil (Isoptera: Rhinotermitidae). Insect Systematics y Evolution 31 (4): 463-472.

CONSTANTINO, R. 2002a. An illustrated key to Neotropical termite genera (Insecta: Isoptera) based primarily on soldiers. Zootaxa 67: 1-40.

CONSTANTINO, R. 2002b. The pest termites of South America: Taxonomy, distribution and status. Journal of Applied Entomology 126 (7-8): 355-365.

CONSTANTINO, R.; ACIOLI, A. N. S. 2006. Termite diversity in Brazil (Insecta: Isoptera). pp. 117-128. En: Moreira, F.; Siqueira, J.; Brussaard, L. (Eds.). Soil biodiversity in Amazonian and other Brazilian ecosystems. Wallingforg: CAB International. $304 \mathrm{p}$.

CONSTANTINO, R.; DE SOUZA, O. F. F. 1997. Key to the soldiers of Atlantitermes Fontes 1979. with a new species from Brazil (Isoptera Termitidae Nasutitermitinae). Tropical Zoology 10 (2): 205-213.

CONSTANTINO, R.; SCHLEMMERMEYER, T. 2000. Estudos previos sobre invertebrados na area de influencia do APM Manso. pp. 129-151. En: Alho, C. J. R. (Coord.). Fauna silvestre da regiao do Rio Manso - MT. Brasilia: IBAMA ediciones. $267 \mathrm{p}$.

CORNELIUS, M. L.; OSBRINK, W. L. 2011. Effect of seasonal changes in soil temperature and moisture on wood consumption and foraging activity of Formosan subterranean termite (Isoptera: Rhinotermitidae). Journal of Economic Entomology 104 (3): 1024-1030.

CORTÉS-PÉREZ, F.; DUEÑAS-GÓMEZ, H. del C.; CARDOZO, H. 2005. Cambios en la vegetación de sabana ocasionados por la plantación de Pinus caribaea en Vichada-Colombia. Revista Academia Colombiana de Ciencias Exactas, Físicas y Naturales 29: 69-84.

CRUZ, A. 1988. Avian resource use in Caribbean pine plantation. The Journal of Wildlife Management 52 (2): 274-279.

CUEZZO, C.; CANCELLO, E. M. 2009. A new species of Obtusitermes (Isoptera: Termitidae, Nasutitermitinae) from South America. Zootaxa 1993: 22-30.

DECAËNS, T.; JIMÉNEZ, J. J.; RANGEL, A. F.; CEPEDA, A.; MORENO, A. G.; LAVELLE, P. 2001. La macrofauna del suelo en la sabana bien drenada de los Llanos Orientales. pp. 111-137. En: Rippstein, G.; Escobar, G.; Motta, F. (Eds.). Agroecología y biodiversidad de las sabanas en los llanos orientales de Colombia. Cali, Colombia: Centro Internacional de Agricultura Tropical. $308 \mathrm{p}$.

DECÄENS, T.; LAVELLE, P.; JIMÉNEZ, J. J.; ESCOBAR, G.; RIPPSTEIN, G.; SCHNEIDMADL, J.; SANZ, P. HOYOS.; THOMAS, R. J. 2003. Impacto del uso de la tierra en la ma- crofauna del suelo de los Llanos Orientales de Colombia. pp. 21-45. En: Jiménez, J.; Thomas, R. (Eds.). El arado natural: las comunidades de macroinvertebrados del suelo en las savanas neotropicales de Colombia. Cali, Colombia. Centro Internacional de Agricultura Tropical. 439 p.

DE CAMINO, R.; BUDOWSKI, G. 1998. Impactos ambientales de las plantaciones forestales y medidas correctivas de carácter silvicultural. Revista Forestal Centroamericana 22: 6-12.

DIBOG, L.; EGGLETON, P.; NORGROVE, L.; BIGNELL, D. E. 1999. Impacts of canopy cover on soil termite assemblages in a agrosilvicultural system in southern Cameroon. Bulletin of Entomological Research 89 (2): 125-132.

EGGLETON, P.; TAYASU, I. 2001. Feeding groups, lifetypes and the global ecology of termites. Ecological Research 16 (5): 941 960.

EGGLETON, P.; BIGNELL, D. E.; SANDS, W. A.; WAITE, B.; WOOD, T. G.; LAWTON, J. H. 1995. The species richness of termites (Isoptera) under differing levels of forest disturbance in the Mbalmayo Forest Reserve, southern Cameroon. Journal of Tropical Ecology 11 (1): 85-98.

EMERSON, A. E.; BANKS, F. A. 1957. Five new species and one redescription of the Neotropical genus Armitermes Wasmann (Isoptera, Termitidae, Nasutitermitinae). American Museum Novitates 1841: 1-17.

FAVILA, M. E; HALFFTER, G. 1997. The use of indicador groups for measuring biodiversity as related to community structure and function. Acta Zoológica Mexicana 72: 1-25.

FERNÁNDEZ MÉNDEZ, F.; CAMARGO MARTÍNEZ, Y. K.; SARMIENTO, M. B. 2012. Biodiversidad vegetal asociada a plantaciones forestales de Pinus caribaea Morelet y Eucalyptus pellita F. Muell Establecidas en Villanueva, Casanare, Colombia. Revista Facultad Nacional de Agronomía, Medellín 65 (2): 6749-6764.

FONTES, L. R. 1982. Novos táxons e novas combinações nos cupins nasutos geófagos da região Neotropical (Isoptera, Termitidae, Nasutitermitinae). Revista Brasilera de Entomología 26 (1): 99-108.

FONTES, L. R. 1986. Two new genera of soldierless Apicotermitinae from the Neotropical region (Isoptera, Termitidae). Sociobiology 12: 285-297.

FRANCIS, J. K.; FOREST SERVICE, N. O. 1992. Pinus caribaea Morelet Pino caribeño. General Technical Report-Forest Service. Southern Forest Experiment Station (USDA). SO-ITFSM-53. 1: 394-403.

GONÇALVES-PERALTA, R. C.; BARSANULFO-MENEZES, E.; CARVALHO, A. G.; AGUIAR-MENEZES, E. D. 2004. Wood consumption rates of forest species by subterranean termites (Isoptera) under field conditions. Revista Árvore 28: 283-289.

GONZÁLEZ, G.; ZOU, X.; BORGES, S. 1996. Earthworm abundance and species composition in abandoned tropical croplands: comparisons of tree plantations and secondary forest. Pedobiología 40: 385-391.

HOUSTON, W. A.; WORMINGTON, K. R.; BLACK, R. L. 2014. Termite (Isoptera) diversity of riparian forests, adjacent woodlands and cleared pastures in tropical eastern Australia. Austral Entomology 54 (2): 221-230.

JAMIL, K. 2001. Bioindicators and biomarkers of environmental pollution and risk assessment. Science Publishers. Michigan, EE.UU. 201 p.

JONES, D. T.; VERKERK, H. J.; EGGLETON, P. 2005. Methods for sampling termites. pp. 221-254. En: Leather, S. R. (Ed.). Insect sampling in forest ecosystems. Ascot: Blackwell Publishing. $303 \mathrm{p}$.

JUNQUEIRA, L.; DIEHL, E. 2009. Termite (Isoptera) diversity in Eucalyptus - growth areas and in forest fragments. Sociobiology 53 (3): 805-828.

JUNQUEIRA, L. K.; DIEHL, E.; BERTI FILHO, E. 2012. Termites in eucalyptus forest plantations and forest remnants: an ecological approach. Bioikos 22 (1): 3-14. 
KRISHNA, K. 1961. A generic revision and phylogenetic study of the family Kalotermitidae (Isoptera). Bulletin of American Museum of Natural History 122: 303-408.

LAVELLE, P.; BIGNELL, D.; LEPAGE, M.; WOLTERS, W.; ROGER, P.; INESON, P.; HEAL, O. W.; DHILLION, S. 1997. Soil function in a changing world: the role of invertebrate ecosystem engineers. European Journal of Soil Biology 33 (4): 159-193.

LAVELLE, P.; DECAËNS, T.; AUBERT, M.; BAROT, S.; BLOUIN, M.; BUREAU, F.; MARGERIE, P.; MORA, P.; ROSSI, J.-P. 2006. Soil invertebrates and ecosystem services. European Journal of Soil Biology 42: S3-S15.

LIMA, J. T.; COSTA-LEONARDO, A. M. 2007. Recursos alimentares explorados pelos cupins (Insecta: Isoptera). Biota Neotropica 7 (2): 243-250.

MALEQUE, M. A.; MATEO, K.; ISHIII, H. T. 2009. Arthropods as bioindicators of sustainable forest management, whit focus on plantation forest. Applied Entomology and Zoology 44 (1): $1-11$.

MATHEWS, A. G. A. 1977. Studies on termites from the Mato Grosso State, Brazil. Academia Brasilera de Ciencias, Rio de Janeiro. 267 p.

NOIROT, C.; DARLINGTON, J. P. 2000. Termites nest: architecture, regulation and defence pp. 121-139. En: Abe, T.; Bignell, D. E.; Higashi, M.; (Eds.). Termites: evolution, sociality, symbioses, ecology. Springer Science y Business Media. 466 p.

NOBRE, T.; NUNES, L.; BIGNELL, D. E. 2009. Survey of subterranean termites (Isoptera: Rhinotermitidae) in a managed silvicultural plantation in Portugal, using a line-intersection method (LIS). Bulletin of Entomological Research 99 (1): 11-21.

OLIVEIRA, D. E. 2013. Sistemática do grupo Velocitermes (Isoptera, Termitidae, Nasutitermitinae). Ph. D. Tesis. Universidad de Brasilia, Brasilia. 274 p.

PEARCE, M. J.; WAITE, B. S. 1994. A list of termite genera (Isoptera) whit comments on taxonomic changes and regional distribution. Sociobiology 23: 247-263.

PINZÓN, O. P.; HERNÁNDEZ, A. M.; MALAGÓN, L. A. 2012. Diversity of termites (Isoptera: Termitidae, Rhinotermitidae) in rubber-tree plantations in Puerto López (Meta, Colombia). Revista Colombiana de Entomología 38 (2): 291-298.
POLATTO, L; ALVES-JUNIOR, V. 2009. Distribuição e densidade de Nasutitermes sp. (Isoptera: Termitidae) em Mata Ribeirinha do Rio Miranda, Pantanal Sul-Matogrossense, Brasil. EntomoBrasilis 2: 27-30.

REGENT INSTRUMENTS INC. 2012. Image analysis for plant science: WinCam Versión 2012a. http://www.regentinstruments.com/assets/wincam_mostrecent.html.

REFOCOSTA S. A. 2013. Resumen plan forestal 2013 - 2020. REFOCOSTA SAS. Bogotá D. C., Colombia 32 p.

R CORE TEAM. 2013. R: A language and environment for statistical computing. $\mathrm{R}$ foundantion for statistical computing. Vienna, Austria. Disponible en: http://www.R-project.org/. [Fecha revisión: 23 febrero 2017].

RINCÓN, S. A. 2006. Recomendaciones sobre biodiversidad a la guía de manejo ambiental para el sector producción primaria del cultivo de caucho natural. Instituto de Investigaciones. Bogotá, Colombia. $17 \mathrm{p}$.

ROCHA, M. M.; CANCELLO, E. M.; CARRIJO, T. F. 2012. Neotropical Termites: revision of Armitermes Wasmann (Isoptera, Termitidae, Syntermitinae) and phylogeny of Syntermitinae. Systematic Entomology 37 (4): 793-827.

SIMBERLOFF, D.; NUÑEZ, M. A.; LEDGARD, N. J.; PAUCHARD, A.; RICHARDSON, D. M.; SARASOLA, M.; VAN WILGEN, B. W.; ZALBA, S. M.; ZENNI, R. D.; BUSTAMANTE, R.; PEÑA, E.; ZILLER, S. R. 2009. Spread and impact of introduced conifers in South America: Lessons from other southern hemisphere regions. Austral Ecology 35 (5): 489-504.

Recibido: 30-nov-2016 • Aceptado: 20-nov-2017

Citación sugerida:

BELTRÁN-DÍAZ, M. A.; PINZÓN-FLORIÁN, O. P. 2018. Termitofauna (Isoptera: Termitidae, Rhinotermitidae) en plantaciones de Pinus caribaea en sabanas de la Orinoquia Colombiana. Revista Colombiana de Entomología 44 (1): 61-71. Enero - Junio 2018. 